\title{
PSYCHOLOGIE ET PSYCHOÉDUCATION
}

Jeannine Guindon ${ }^{12}$

RÉSUMÉ: La reconnaissance de la psychoéducation par la Faculté des Arts et des Sciences de l'Université de Montréal comme une nouvelle discipline a demandé d'établir clairement ses différences avec la Psychologie. La présentation orale qui a servi à en faire la preuve en 1971 devant le conseil de cette Faculté est transcrite ici dans un caractère plus gros. Le texte rédigé dans un caractère plus petit ne faisait pas partie des explications offertes aux universitaires. Il est ajouté par la rédaction de la revue pour aider les lecteurs à saisir davantage la signification de la présentation tout en précisant des éléments du cadre de référence de la psychoéducation qui ont continué d'évoluer dans la lancée du début avec la recherche et l'observation de l'expérience.

Mots Clés: Psychoéducation, concept differentiel, Psychologie

\section{PSYCHOLOGY AND PSYCHOEDUCATION}

ABSTRACT: The recognition of Psychoeducation by the Faculty of Arts and Sciences of the Montreal University as a new discipline occured after distinction with Psychology was clearly established. The oral presentation that gave support to prove this in 1971 in front of the Faculty Counsil is now presented here, in its general format. This new text, written with all its details, isn't the same offered to the students in the university. The explanations added by the journal have the objectif of showing all the importance of this presentation and so the context of Psycholeducation is given, even knowing that its evolution continues due in part to research and experience observation.

Key words: Psychoeducation, conceptualization differential, Psychology

Par le présent texte, nous voulons:

1) En réponse à une demande expresse du Département de Psychologie, établir clairement la distinction entre les deux disciplines: Psychologie et Psychoéducation;

2) Faciliter l'étude du programme de maîtrise en Psychoéducation.

Des remarques préliminaires assez élaborées s'imposent:

Vingt années d'efforts constants nous ont permis d'arriver à formuler les concepts théoriques et cliniques qui sous-tendent le processus rééducatif

\footnotetext{
${ }^{1}$ Artigo orignalmente publicado pelo SANS Frontières, revista do Centre de Recherche de l'Institut de Formation Humaine Intégrale de Montréal

${ }^{2}$ Endereço para correspondência : Jeannine Guindon, Institut de Formation Humaine Intégrale de Montréal, 55, Boulevard Gouin Ouest, Montréal, Québec, H3L 1H9. e-mail ifhim@total.net.
}

Paidéia, FFCLRP-USP, Rib. Preto, junho/99. appliqué par les psychoéducateurs cliniciens auprès d'enfants, d'adolescents et d'adultes inadaptés.

La formulation de ces concepts provient:

a) D'une analyse rétroactive constante des données et des faits recueillis quotidiennement par des équipes de psychoéducateurs en cours de pratique professionnelle;

Une analyse rétroactive demande d'abord de faire l'expérience pour ensuite l'analyser. Les psychoéducateurs étudiaient les faits et arrivaient à une conceptualisation. Ils procédaient par induction à partir des faits. Ainsi la thèse doctorale de Jeannine Guindon intitulée 'Le processus de rééducation du jeune délinquant par l'actualisation des forces du moi' s'est élaborée au cours de plusieurs années. L'étude s'opérait avec des enfants, des adolescents et des adultes inadaptés, parents des adolescents. 
b) De l'apport d'équipes pluridisciplinaires qui, voulant cerner de près cette conceptualisation d'un processus de rééducation en voie de développement, ont étudié attentivement ces observations recueillies;

Ces équipes multidisciplinaires complétaient l'équipe des psychoéducateurs pour étudier les cas traités. On y retrouvait psychiatres, psychologues, psychanalystes et sociologues.

c) Des découvertes constamment suscitées par une étude suivie dans le champ de la psychoéducation.

Ce qui s'opérait dans le champ de la psychoéducation demandait à être précisé cliniquement. Les découvertes constamment suscitées par l'étude se faisaient par induction et déduction. D'autres se faisaient en cours de pratique et les professionnels se rendaient compte que ces observations pouvaient aider les jeunes.

La poursuite de ces recherches appliquées nous a acheminés graduellement vers une réflexion épistémologique propre à ce champ d'étude, pour aboutir à la formulation d'une nouvelle discipline: la Psychoéducation. Cette discipline repose principalement sur les Sciences Psychosociologiques.

Les psychoéducateurs avaient à préciser les connaissances propres à leur champ d'étude et à élaborer des nouveaux concepts. Ils devaient être capables de le faire dans le champ de la psychoéducation qui exigeait une autre façon de structurer et d'organiser la connaissance.

En plus de reposer sur la Psychologie, la Psychoéducation s'appuie aussi sur la Sociologie. Elle tient compte de l'étude du milieu et des interactions entre les générations, cherchant à voir comment les personnes sont affectées ou soutenues par leur entourage. La situation des jeunes en difficulté qui étaient traités demandait une étude du milieu.
Son champ d'application exige un cadre de référence théorique intégré qui d'une part, envisage le développement humain dans un sens épigénétique et d'autre part, suppose une conceptualisation de l'entourage.

L'éducation s'inspire de différentes formes de conceptualisation mais ne présente pas de cadre de référence théorique unifié qui intègre les divers éléments du développement. Le président du conseil des universités qui avait lu la thèse de J. Guindon sur la rééducation du jeune délinquant connaissait sa façon de conceptualiser et y voyait une vision pour l'avenir. Cette dernière reçut de l'université le mandat de chercher à préciser le cadre de référence théorique des Sciences de l'Éducation.

Les psychoéducateurs partaient de l'enfant, de l'adolescent et de l'adulte. Mais ils devaient aussi tenir compte des changements qui avaient lieu dans l'entourage, à travers les époques historiques et l'évolution sociogénétique ${ }^{1}$

Le psychoéducateur clinicien est forcé de recourir à une conceptualisation théorique qui sauvegarde l'unité même de la personne insérée dans un contexte de vie (contexte historicogéographique, économicopolitique et socioculturel). Il abordera l'étude de l'individu dans son développement intégral: physique, affectif, cognitif, social et religieux (épigenèse intégrale), en tenant compte de son interaction avec son milieu de vie dans lequel il évolue. II considérera donc cet individu comme une entité qui recherche son unité dans le contexte socioculturel québécois, selon le processus historique actuel (changements rapides) et le

\footnotetext{
${ }^{1}$ Cette conceptualisation de l'entourage a fait un pas important avec la mise au point des différentes facettes nécessitées par un engagement social dans un projet collectif. Ce processus élaboré par M.-M. desmarais à l'intérieur du cadre de référence de l'actualisation des forces vitales humaines vise à respecter la place de chacun dans un projet qui tend à operer des transformations dans le milieu. Lire dans La Vie sans frontières, mai 1992, IFHIM, Montréal, p. 31-38.
} 
contexte géographique, économique et politique qui est le nôtre. Le psychoéducateur clinicien doit recourir à des théories et des méthodes qui offrent à l'individu « a whole world to be whole in ».

«A whole world to be whole in »: un monde avec ses multiples facettes où l'individu peut arriver à se sentir unifié. Parvenu à une certaine autonomie psychique, l'individu peut faire un retour sur son passé en se servant de sa vision différenciée du présent. Avec ses acquis, il peut voir son histoire passée avec les yeux du présent et faire les différences. Autrement, ce retour risque de traduire une nostalgie et de constituer un refus du présent, ce qui nuit à l'engagement et à l'actualisation des forces vitales.»

Une telle systématisation d'un cadre de référence théorique a été élaborée principalement sous l'inspiration de plusieurs auteurs de différentes écoles de Psychologie tant psychanalytique que génétique et académique. Tous sont d'accord sur un concept: celui de l'épigenèse. De ces multiples apports théoriques ressortent plus particulièrement les concepts d'Erikson' (analye du moi de l'école psychanalytique) qui englobent l'apport de Piaget $^{3}$ pour rendre compte de l'épigenèse des structures cognitives et permettent une conception qui, tout en respectant des données psychanalytiques de forces instinctuelles, intègre le point de vue de l'apprentissage à la formation des structures intrapsychiques dans l'individu. De

\footnotetext{
${ }^{2} \mathrm{La}$ formation d'Erikson comme anthropologue lui a beaucoup servi dans l'élaboration de ses concepts intégrés.

${ }^{3}$ Jean Piaget $<$ considère que les empiristes positivistes, qui croient que la connaissance des faits physiques découle de l'ex périence immédiate, des sensations, sont dans l'erreur, car le processus psychologique qui intervient dans toute connaissance n'est pas une association de perceptions, ni même une «association» du type «stimulus-réponse», mais bien une intégration, une incorporation, une «assimilation» de l'objet, à partir d'une action sur lui. En jouant avec un objet, l'enfant agit sur lui et le transforme (ne serait-ce qu'en modifiant sa position). Pour Piaget, toute connaissance résulte d'une construction qui dépend elle-même d'un mécanisme psychobiologique de régulation entraînant la création continuelle de structures nouvelles.» (Sillamy, p. 517)
}

Paidéia, FFCLRP-USP, Rib. Preto, junho/99. plus, l'apport psychosocial de ce théoricien, qui est anthropologue autant que psychologue, fournit une certaine conceptualisation de l'entourage qui facilite l'étude de l'évolution sociogénétique du milieu, la séquence des générations et la structure de la société en relation avec le processus épigénétique intégral de l'individu: c'est-à-dire les formes, les moeurs, les traditions, le mode de vie et les institutions sociales.

Pour mieux étayer les distinctions qui s'imposent, il serait utile de recourir à quelques considérations apportées par Jean Piaget sur les problèmes épistémologiques actuels. Il souligne la présence de « deux grands groupes de méthode » pour aborder ce problème de la connaissance ${ }^{4}$.

« Les unes sont aprioriques ou essentiellement réflexives et partent de la présupposition que les principes de la connaissance peuvent être atteints directement par une discipline autonome et de rang supérieur, dont les résultats permettraient alors un contrôle normatif sur les sciences particulières ou sur les connaissances en leur diversité ».

«Les méthodes du second groupe partent au contraire de l'idée que les connaissances comportent des modes de structuration imprévisibles, multiples et sans cesse renouvelés et que leur analyse ne peut alors procéder avec fruits qu'après constitution ou au cours de leur création; et autant que possibie de l'intérieur, avec le minimum de présuppositions, celles-ci courant toujours le risque d'être déformantes ».

Piaget montrait que les méthodes de ce second groupe avait une validité au niveau de la connaissance. Dans le cadre de référence des forces vitales humaines par l'observation de l'expérience, la Psychoéducation a vérifié et continue de vérifier que la connaissance a des modes de structuration imprévisibles, multiples et sans cesse renouvelés. A titre d'exemple, voyons l'évolution des découvertes

\footnotetext{
${ }^{4}$ Préface du volume <<Logique et connaissance scientifique〉>, sous la direstion de Jean Piaget, Encyclopédie de la Pléiade, 1969
} 
cliniques et théoriques qui ont conduit à la formulation du concept de l'identité de soi. En 1969, on parlait des étapes (acclimatation, contrôle, production, personnalité). En 1979, on décrit les quatre axes comme des angles de vision du vécu de la personne (corps vécu, productivité, adaptabilité, rencontres significatives). Les sois sont présentés en 1982 comme les éléments constitutifs de "la représentation de soi en interaction avec la représentation de l"entourage et des autres" (soi corporel, soi productif, soi adaptatif, soi social). La recherche a permis d'en arriver en 1985 au concept de l'identité de soi qui, en lien avec le sens qu'une personne donne à sa vie, unifie les facettes de la personnalité (corporelle, d'exécutant, individuelle, psychosexuelle, psychosociale). L'usage des concepts dans l'expérience suscite cette évolution conceptuelle continuelle qui permet ensuite de s'approcher de l'expérience avec un équipement plus adéquat et de rester attentif à la vie toujours en mouvement. $^{5}$

En un mot, selon ees méthodes, la question n'est que de chercher à comprendre comment il a procédé ou pourquoi il a mieux réussi en certaines occasions qu'en d'autres.

Dans le cadre de référence des forces psychologiques, l'intervenant observe la façon dont la personne a procédé. L'accent est mis sur le comment et sur ce qui fait $q u$ 'elle a réussi. La Psychoéducation se sert donc d'une épistémologie propre, différente de celle des sciences pures. Elle part de l'observation de l'expérience.

"Tous les courants vivants de l'épistémologie contemporaine font aujourd'hui corps avec les sciences elles-mêmes, en ce sens que les transformations si imprévues et souvent si rapides de diverses disciplines ont entraîné des crises et des réorganisations obligeant les savants à examiner les conditions mêmes de leur savoir, donc en fait à construire des épistémologies".

\footnotetext{
s voir Ia revue SANS FRINTIÈRES les fordes psychologiques, automne 193, 8(2), p.4.
}

Ĺa façon de penser jusque là partait du principe qu'il n'y avait qu'une seule épistémologie et, selon cette façon de voir, la Psychoéducation n'était qu'une branche de la Psychologie. Piaget affirme que chaque science a sa méthode de recherche et de connaissance. Observer l'expérience pour voir comment la personne a procédé et comment elle a réussi est très différent de la méthode qui consiste à faire une hypothèse et à vérifier son application. La Psychoéducation veut savoir comment, ce qui est différent de la façon de faire dans une science pure appliquée.

\begin{abstract}
"En d'autres termes, le problème de "fondements" n'est plus réservé à une discipline extérieure à la science comme telle, ainsi que ce pouvait être le cas à une époque ou la pérennité supposée des principes entretenait une douce quiétude et rendait inutile cet effort constant d'une analyse rétroactive et de réflexion épistémologique auquel les créateurs scientifiques sont aujourd' hui conduits de façon irrésistible par le mouvement même de leur recherche proactive et de leurs découvertes".
\end{abstract}

Le champ d'études de la Psychoéducation se réclame de ce second groupe de méthodes. Dans l'optique de la Psychoéducation, la plupart des travaux concrets sont d'inspiration structuraliste génétique, à savoir:

1- Que toute vie psychique est étroitement liée à la praxís;

On ne peut pas abstraire la vie psychique de l'expérience, de la praxis. Toute vie psychique demeure étroitement liée à l'expérience.

2- Qu'elle se présente ensuite sur le plan individuel comme sur le plan collectif sous la forme de réalités dynamiques orientées vers un équilibre cohérent entre le sujet et le milieu ambiant processus qu'il est convenu d'appeler "processus de structuration". Ce processus de structuration comporte des ensembles dont les parties constitutives sont interdépendantes et liées entre

Paidéia, FFCLRP-USP, Rib. Preto, junho/99. 
elles par des règles spécifiques et des possibilités limitées de transformations.

Le dictionnaire Le petit Robert parle de la structure comme d'un agencement de différentes facettes. Elle est une organisation stable. Le "processus de structuration" mène à une unité fonctionnelle et structurale. Piaget et Erikson sont d'accord sur ce concept.

Pour le décrire, la capacité de gérer ses énergies physiques et psychiques peut servir d'exemple. Si quelqu'un est en mesure de le faire de façon ajustée, on peut parler d'équilibration.

Il peut prendre les décisions appropriées pour parvenir à cet équilibre et vivre en unité. Si l'expérience se maintient et s'intègre, l'individu parvient à une intériorisation. Il se l'approprie et devient capable de plus en plus de conserver cet équilibre même quand les conditions changent et que surviennent des imprévus. On peut parler alors d'unité structurelle.

L'individu développe l'amour de soi en devenant capable de prendre des décisions qui sont appropriées et en accord avec son objectif de garder son équilibre et de vivre en harmonie avec lui-même et avec les autres. Il refait plusieurs fois l'expérience, la relit et réfléchit. Il peut ainsi en arriver à la conserver et à l'intérioriser de telle sorte qu'elle en vient à faire partie intégrante de son organisation psychique. Ceci facilite ensuite la mise en action des moyens à maintenir pour conserver ou retrouver l'équilibre des énergies. Cet apprentissage devient une structure stable et intégrée dans l'existence de l'individu.

Quand cette structure fait partie de la vie de l'individu et s'accompagne d'une cohérence avec les décisions mises en acte, on peut parler d'organisation fonctionnelle. Cette dernière se manifeste par l'actualisation des forces vitales dans des décisions appropriées. Cette unité structurale apparaît alors dans une consolidation vérifiée par une stabilité Paidéia, FFCLRP-USP, Rib. Preto, junho/99. temporelle, une cohésion structurale et une coloration affective positive.

Des nouvelles structures se développent même chez l'adulte. Elles dépassent les niveaux d'organisation psychique mis en place de la naissance à l'adolescence. On parle alors de processus de structuration initié dans un premier temps par une actualisation des forces vitales qui mène à une structuration de la personnalité. Les changements qui demeurent indiquent la mise en place d'une structure psychique permanente. Malgré les changements de son entourage physique et humain, l'individu continue de maintenir ses ajustements en trouvant, ou même en créant au besoin, des façons de faire et des moyens qui lui permettent de conserver ou de retrouver son equilibre physique et psychique. La personne refait son equilibre intérieur et maintient son harmonie où qu'elle soit.

Le texte affirme: "Les parties constitutives sont interdépendantes et liées entre elles par des règles spécifiques et des possibilités limitées de transformation". Les différents éléments de la personne sont interreliés comme des facettes. Par exemple, si quelqu'un ne développe pas une autonomie corporelle, ses autres décisions s'en ressentent. Telle que précisée à partir de l'observation, la règle spécifique du développement est épigénétique. Ce dernier s'opère à la façon d'une spirale oú les acquis d'une étape s'appuient sur la précédente et préparent la suivante. Les acquis doivent en venir à faire partie de l'individu. Autrement il peut faire des changements mais ils ne seront pas stables, parce que non intégrés. Il n'y a alors ni harmonie, ni structuration. Il est possible d'observer quand certaines personnes sont arrivées à un niveau de transformation qui va demeurer et d'autres dont l'équilibre dépend du soutien de l'entourage oú elles se retrouvent. La stabilité temporelle demande que soit établie une structuration de base sur laquelle le reste de l'édifice peut s'édifier. 
Le progrès de la croissance d'une structure globale entraîne le plus souvent la possibilité de mieux connaître et comprendre ses éléments, alors qu'inversement, Je progrès de la connaissance de ces éléments permet de revenir de manière opératoire à l'ensemble.

Dans son livre sur la rééducation du jeune délinquant par l'actualisation des forces du moi, (p.289-290), Guindon précise que "Selon l'étape de rééducation atteinte, et à chacune des étapes, l'acquísition des composantes partículières de ces vertus psychologiques se manifestera chez le jeune par une certaine unité fonctionnelle de Ia personnalité, pour culminer en une «unité structurale», aussí appelée structure d'ensemble, caractéristique de l'étape en cours. Vers la fin du processus de rééducation, une certaine unité devra s'être manífestée dans la personnalíté du jeune homme, unité qui se consolidera dans la formation d'une identité positive.

"En présentant une synthèse qui a voulu tenír compre aussi bien de l'ensemble du processus de rééducation du jeune délinquant que des éléments qui le composent, cette étude n'a faít qu'esquisser en gros traíts /e problème tellement complexe de ce processus, maís el/e aura tout de même permis de transmettre une expérience accumulée grâce à une étude systématíque des données relatives à ce domaine recueillies jusqu'à présen".

L'unité fonctionnelle et structurale sont des structures globales. Le processus de formation humaine intégrale est perçu aussi comme une structure d'ensemble composée des diverses facettes de l'identité de soi. Ces facettes se construisent par l'actualisation des forces vitales humaines. Plus la personne avance dans la connaissance d'une structure globale, plus elle en perçoit les éléments. Plus elle différencie chez elle le processus de formation humaine intégrale et plus elle précise les divers éléments qui le composent par leur interaction. Et l'inverse est aussi vrai. L'interaction des divers éléments lui fait voir les liens qui les unissent et la ramène à l'ensemble. Ainsi, plus elle saisit le sens et l'importance de l'identité corporelle, plus elle perçoit le rôle que joue cette dernière et les répercussions exercées dans les relations avec les autres, la sexualité, la contribution et les façons de faire. Le terme opératoire indique les liens logiques perçus entre les divers éléments face à l'ensemble. Les découvertes vont dans les deux sens: des éléments à l'ensemble et de l'ensemble aux éléments. Au moment de cette présentation en 1971 à I'Université de Montréal, le concept de représentation n'était pas encore formulé, mais tout le sens de ce conceptcharnière était déjà présent. Il represente le fondement de cette nouvelle discipline de la Psychoéducation.

Le champ d'étude de la Psychoéducation, ayant systématisé un cadre de référence théorique intégré, adopte le point de vue du "structuralisme relationnel" pour trouver les modèles de construction progressive des structures d'ensemble qui formalisent, d'une part, l'épigenèse intégrale de l'individu (stades, étapes) et d'autre part, l'évolution sociogénétique du milieu.

Le point de vue adopté, c'est-à-dire le structuralisme en tant que relationnel, se définit selon les termes de Piaget :

« (...) en tant que posant à titre de réalité première les systèmes d'interactions ou de transformations subordonnant donc dès le point de départ les éléments à des relations qui les englobent et concevant réciproquement le tout comme le produit de la composition de ces interactions formatrices. »

Ce passage et la citation de Piaget reprennent les termes de la conclusion de la thèse de Guindon (p. 290). Cette dernière avait découvert dans les faits comment les divers

Paidéia, FFCLRP-USP, Rib. Preto, junho/99. 
éléments d'une structure d'ensemble s'interreliaient. Piaget venait à ce moment de préciser ce qu'il entendait par structuralisme relationnel. Ce qu'il énonçait de façon théorique correspondait aux conclusions des observations faites dans l'expérience.

L'auteure s'en est servi pour expliquer sa conclusion en précisant ce qu'elle entendait par le concept de « structure »: «À l'intérieur de cette synthèse a aussi été abordé le problème si difficile de «vouloir caractériser la vie affective par rapport aux fonctions cognitives (en autant que celles-ci sont relatives aux structures) et surtout à vouloir préciser leurs relations dans le fonctionnement même des conduites" conduites observées dans le comportement quotidien.

«Il a été envisagé différemment de la tendance générale qui a cours aujourd'hui et qui veut distinguer en toute conduite, une structure qui correspondrait à son aspect cognitif et une «énergétique» qui caractériserait son aspect affectif»

Le concept de «structure» a donc été pris dans un sens beaucoup plus large afin de tenir compte, non seulement de l'aspect cognitif mais aussi du développement des forces vitales et de l'Ego, cet agent central de la formation de la personnalité - agent cohérent et persistant qui sélectionne, intègre et unifie les motivations, les pensées et les actes de l'individu».

À titre de réalité première, comme dans une approche systémique, les éléments ne sont pas isolés et trouvent une signification en autant qu'ils sont dans un système d'interactions ou de transformations subordonnant dès le point de départ les éléments à des relations. Par exemple, le concept d'identité comprend cinq facettes indissociables: l'identité d'exécutant ne peut se séparer de l'identité corporelle, ni l'identité individuelle de l'identité corporelle et d'exécutant; de même l'identité psychosexuelle ne peut se détacher des autres non plus que l'identité psychosociale. Cela fait de l'identité de soi un système d'ensemble. Les systèmes d'interactions ou de transformations font que, pour transformer l'identité psychosexuelle, il faut transformer l'identité corporelle, l'identité d'exécutant et les autres facettes. À titre de réalité première, ces transformations subordonnent dès le point de départ les éléments à des relations. Si l'un d'eux se transforme, les autres se transforment aussi. On pourrait prendre également comme exemple l'interaction entre les forces vitales humaines.

Ce structuralisme relationnel fournit des lois de construction au lieu de s'en référer d'une part, à une totalité conçue comme toute faite (la Gestalt), ou, d'autre part, de réduire les phénomènes à des éléments atomistiques dont la somme des propriétés rendrait compte du total à interpréter (les théories associationnistes de l'apprentissage de Hull).

La Gestalt représente une perception globale qui est tout organisée. ${ }^{6}$

«D'après les tenants de la théorie de la forme (Gestalttheorie), les formes sont des données premières, des unités perceptives qui s'imposent à la conscience. » Le cadre de référence des forces vitales traite du développement, de croissance, d'étapes, de construction. Les identités, les forces et les niveaux d'organisation psychique se construisent.

Réduire les phénomènes à des éléments atomistiques consiste à séparer les éléments

\footnotetext{
' «La psychologie de la forme (Gestalpsychologie), de l'école de Berlin, qui se constitua peu avant la Première Guerre mondiale autour de Max Wertheimer (1880-1943), en réaction aux théories atomistes et mécanicistes de la psychologie associa tionniste classique, a montré que le monde dans lequel nous vivons ne nous est pas donné sous forme de sensations élé mentaires, indifférenciées, que notre cerveau organiserait, mais est composé de formes significatives. Celles-ci ne sont pas apprises, mais appréhendées dès le premier instant en tant que Gelstalt.» (Sillamy, p.285)
} 
les uñs des autres comme dans le cas du béhaviorisme ${ }^{7}$. Il n'est cependant pas possible de parvenir à connaître un tout, simplement par la connaissance des propriétés de chacun des éléments. Cela demande de percevoir les interrelations entre chacun des éléments. Le béhaviorisme présente des ressemblances avec l'associationnisme. Clark Leonard Hull (1884-1952) était alors un psychologue très connu sur les theories d'apprentissage. Il est l'une des principales figures de la première génération américaine des néo-béhavioristes. «Après les années cinquante, le système de Hull cessa d'être pris au sérieux, car on découvrait de plus en plus de faits importants dont sa théorie ne pouvait rendre compte.(Sillamy, p. 328)» II a adopté certaines vues de l'associationnisme. ${ }^{8}$ pour développer sa théorie. Lui aussi séparait les divers éléments dans le développement. Si quelqu'un fait de la dissection, il peut parler de chacun des éléments du corps humain, mais il ne peut expliquer la vie de cette façon. La somme de chacune des propriétés des éléments expliqués ne rend pas compte du total à interpréter. Associer les éléments atomistiques ne suffit pas pour comprendre le développement humain. Il faut en arriver à percevoir les interrelations transformantes entre chacun des éléments.

\footnotetext{
${ }^{7}$ Le terme a été « introduit par J-B- Watson (1913) pour désigner la doctrine dont il fut le prometeur et selon laquelle l'objet de la psychologie devrait se limiter aux seules données observables du comportement.. En négligeant les faits de conscience et en rejetant catégoriquement l'introspection, le béhaviorisme s'est contraint à n'examiner que les comportements élémen taires et à ignorer les phénomènes complexes tels que les idées, le raisonnement ou les sentiments... Mais la position initiale des béhavioristes est aujourd'hui dépassée. Dès 1929, Hull, rejoint par d'autres, élaborait une théorie compréhensive du comportement dans laquelle l'organisme (ses besoins, ses motivations) était réintégré. (Siliamy, p. 81) 》

${ }^{8} \mathrm{~L}$ 'associationnisme est la « théorie selon laquelle la vie mentale est constituée de chaînes associatives dé faits élémentaires de conscience... Le reproche principal qu'on peut lui faire est de considérer l'organisme comme un être passif, régi par des lois mécaniques, et l'esprit comme un àgrégat d'éléments psychiques se regroupant automatiquement, au lieu de voir son unité, sa permanence et sa structure (Sillamy, p. 61-62). »
}

Le «constructivisme relationnel» ou dialectique par sa double préoccupation de la " totalisation " et de la formation historique est conduit à faire la synthèse entre les considérations de structure et de genèse.

«Constructivisme relationnel», une expression de Piaget, indique une interaction entre les composantes. Dans le domaine de l'apprentissage, il n'y avait en Psychologie à ce moment-là que la théorie de Hull. Guindon explique l'apprentissage différemment des béhavioristes et de Hull qui affirmaient que le développement était continu. Elle parle d'épigenèse, donc de stades dans le développement. Une question se posait. Si l'apprentissage se fait selon des stades, comment démontrer que l'évolution de la personne se fait en même temps selon une ligne continue? L'auteure a expliqué au début de sa thèse de doctorat la conjugaison de la continuité et la discontinuité. La continuité se manifeste dans les forces psychologiques qui tracent la ligne du développement. Depuis, le concept intégré de l'identité de soi a permis d'aller plus loin. Par les forces s'élaborent les facettes de l'identité de soi sans lesquelles la représentation de soi, de l'entourage et des autres ne peut se différencier. Cette dernière $s$ 'opère par les forces dans les identités.

Il fait la coordination des deux perspectives conduisant ainsi à concevoir toute structure comme le produit d'une genèse et toute genèse comme procédant à partir de structures antérieures.

Parler d'épigenèse, c'est parler de structures bâties les unes sur les autres. Ainsi, les niveaux d'organisation psychique constituent des structures et leur genèse s'opère dans l'interaction entre l'enfant, le pourvoyeur de soins et le milieu. Toute genèse suppose une structure antérieure. Après le premier niveau vient le deuxième qui origine du premier et ainsi de suite. Le développement de

Paidéia, FFCLRP-USP, Rib. Preto, junho/99. 
l'adolescent suppose l'élaboration successive des cinq niveaux d'organisation psychique. Ensuite, l'évolution se fait par le passage à travers les phases de la vie adulte qui s'appuient sur le développement précédent. Le processus de croissance humaine intégrale est continu et appuyé sur une base élaborée au cours de stades.

Les phases de la vie adulte ne constituent pas un sixième niveau d'organisation psychique. Construite au cours de l'enfance et de l'adolescence, l'organisation psychique fait place à la croissance continue.

Cinq niveaux mènent la personne à l'orée de la vie adulte. Les phases constituent ensuite un processus d'intériorisation basé sur les facettes de l'identité de soi qui se construit progressivement par les forces. La phase n'est pas une structure permanente comme le sont les niveaux d'organisation psychique. Celui dont le développement s'est déroulé de façon harmonieuse jusqu'à dix-huit ans et qui n'a pas fait de réalisations dans la phase de la vingtaine ne serait pas obligé de refaire toutes les étapes pour en arriver à donner un sens à ses réalisations. Son manque en est un d'expérience et non d'organisation psychique. Arrivé à la trentaine, il peut faire assez vite les réalisations et aller au sens à leur donner sans devoir reprendre tout le cheminement des étapes. Mais il doit avoir vécu certaines réalisations et $y$ avoir découvert un sens pour ensuite pourvoir en donner un à ce qu'il veut vivre. Son niveau d'autonomie psychique lui permet plus ou moins facilement de se rattraper et de laisser émerger la question du sens.

Les lois de construction d'une structure fournie par le «structuralisme relationnel» feront l'objet de recherche en tant que systèmes d'ensemble, comme irréductibles aux propriétés des éléments, d'où la conséquence possible consistant à attribuer à cette structure d'ensemble les caractères d'un fait premier ne s'expliquant que par lui-même.
Les lois de construction d'une structure ne fonctionnent pas avec des éléments séparés mais en interrelation et touchent les transformations des éléments les uns par rapport aux autres. Les facettes de l'identité de soi, de même que les forces vitales sont des exemples de systèmes d'ensemble. Les facettes de l'identité ne peuvent être séparées les unes des autres. Ensemble, elles constituent la personnalité d'un individu. De même pour les forces. On ne peut, par exemple, travailler simplement le temps en l'isolant des indices des autres forces. Les décisions ne sauraient alors être appropriées car le temps serait détaché de l'expérience. Dans la réalité, le temps n'est pas détaché de l'espace, des objectifs et des moyens. Les indices des forces sont en interaction. et ne peuvent être détachés de façon atomistique. C'est un fait premier. L'actualisation des forces vitales est un processus systémique. C'est une des caractéristiques de cette approche.

La méthode d'approche pour interpréter les questions de fondements des concepts psychoéducatifs sera celle de la méthode relationnelle. Celle-ci consiste à n'utiliser aucune entité ou terme à signification absolue, mais à manipuler ou composer uniquement des relations dont les termes demeurent indissociables de ces relations mêmes. En d'autres termes, la méthode relationnelle consiste à ne partir ni d'éléments isolés préalables (méthode atomistique) ni de totalités toutes faites correspondant à des intuitions primitives (comme la Gestalt), mais d'une construction de relations dont chacune est déjà totalisante en un sens et qui aboutissent à des structures d'ensemble ou totalités au sens strict, en les constituant sous une forme intelligible.

Entités et termes sont toujours interreliés dans les concepts psychoéducatifs. Les forces, les identités, les niveaux d'organisation psychique sont des termes indissociables des relations qui existent entre eux. 
L'identité corporelle est totalisante en un sens car elle est rattachée à l'identité de soi et aboutit à une structure d'ensemble. Même si chacune des facettes de l'identité de soi est totalisante, elles ne constituent pas séparément une structure d'ensemble. Travailler seulement l'identité corporelle aide la croissance mais ne suffit pas pour parvenir à une représentation différenciée de soi, de l'entourage et des autres. Au niveau des facettes et de la structure d'ensemble s'opèrent continuellement des transformations. L'interrelation entre les identités compose l'identité de soi, structure d'ensemble. Il importe de voir comment l'identité corporelle, l'identité d'exécutant, l'identité individuelle, psychosexuelle et psychosociale se rattachent chacune à l'identité de soi.

Une forme intelligible suppose que sa construction dans l'expérience peut être expliquée. Les individus qui se forment selon le processus de l'actualisation des forces vitales sont ensuite capables d'expliquer leur cheminement. Le cadre de référence théorique donne les éléments pour percevoir et comprendre la croissance dans leur vie. $\mathrm{Ce}$ processus n'est pas seulement psychologique mais constitue une formation humaine intégrale. La dimension théorique permet d'expliquer ce qui se fait au niveau clinique.

Selon les termes mêmes de Piaget, la méthode relationnelle se caractérise principalement par «la considération primordiale du devenir» (en négation de toute conceptualisation statique). Cette ouverture nécessaire sur le devenir et les dimensions historiques ou génétiques conviennent à la discipline de la Psychoéducation. Cette marche dialectique suppose que chaque nouvel échange entre le sujet et l'objet (ou entre la personne et son entourage) ouvre la perspective d'un nouveau progrès possible soit dans la conquête du réel, soit dans l'affinement des instruments déductifs.

Il s'agit de considération du devenir, de croissance humaine et non de statisme. Plusieurs éléments peuvent être déduits mais la conquête du réel n'est pas oubliée. La relecture, ce moyen employé pour aider la personne à se découvrir et à se représenter, est un instrument déductif qui continue de s'affiner tout autant que la façon de travailler l'anticipation et la planification. La relecture du vécu dans le présent et le passé construit le fondement, établit le préalable. Elle permet d'en venir ensuite à anticiper et planifier le devenir.

La conquête du réel se fera par une objectivation plus poussée, en recourant par voie expérimentale à l'étude de la construction des structures opératoires, en d'autres termes, en reconstituant par expérience sur les niveaux de développement une genèse réelle.

La construction des structures opératoires se réfère aux schèmes opératoires formels, par exemple aux liens et combinaisons faits après avoir observé le développement qui s'opère à tel âge pour en expliquer la genèse. Le chercheur qui tente de vérifier si ses déductions sont justes et concordent avec le réel parvient à une objectivation plus poussée.Ceci a été fait avec les jeunes délinquants du centre de rééducation de Boscoville. Les déductions étaient revérifiées de façon systématique par les observations précises des éducateurs dans les comportements du jeune au sport ou ailleurs. Le recours à la voie expérimentale servait à reconfirmer la justesse des déductions en les refaisant et à atteindre une objectivation plus poussée. Il ne s'agissait pas de croire mais de vérifier dans les faits. Si les faits confirment de nouveau, l'objectivation s'accroît. De cette façon s'élargit la conquête du réel.

L'affinement des instruments déductifs pourra se réaliser en faisant appel aux Sciences Logico-Mathématiques qui peuvent aider à l'axiomatisation des structures opératoires en recourant au parallélisme entre les problèmes 
axiomatiques de structures abstraites et les problèmes génétiques des structures opératoires.

Ce travail qui reste encore à faire visait un programme de doctorat, une des conditions pour ouvrir un département autonome universitaire. Un programme d'avant-garde à ce moment-là a été présenté. Approuvé par l'Université de Montréal, il n'a pas été mis en pratique. Le doctorat en cours est multidisciplinaire.

Guindon voulait mettre au point des mathématiques qualitatives pour faire des hypothèses de recherche. Face à des éléments en interrelation qui se transforment, la Psychoéducation a besoin d'instruments pour saisir le qualitatif et non d'instruments normatifs comme le sont les statistiques par exemple. Mario Bunge, professeur de mathématiques et philosophe des sciences, s'intéressait à ces recherches et voyait une science de l'avenir dans ces formules mathématiques qui peuvent se transformer pour suivre les transformations dans l'évolution des individus. Le plan de doctorat s'intéressait aussi à la cybernétique, aux probabilités météorologiques et à diverses sciences ayant à voir avec des éléments en transformation.

Axiomatiser consiste à organiser sous forme d'axiomes par un système de déduction ou par formalisation. L'axiome se définit comme une vérité indémontrable mais évidente pour quiconque en comprend le sens (principe premier). Les mathématiques qualitatives visaient à ce que les combinaisons faites à la suite des observations permettent d'établir un axiome mathématique en lien avec les problèmes génétiques, pour mieux voir comment se font les transformations. Si un axiome permet de suivre la genèse, il donne lieu à une méthode scientifique. Pour être scientifique, une méthode doit faire appel à une forme mathématique qui tient compte de ce qui est observé. L'expérience rend compte de ce qui est vrai, et non les instruments déductifs.
Le Dictionnaire usuel de Psychologie publié en 1983 affirme que les Sciences Humaines manifestent un intérêt croissant pour les méthodes dites "quantitatives" et pour les modèles mathématiques. Il opine aussi que ceux-ci sont parfois exagérément sophistiqués eu égard à la nature des connaissances expérimentalement contrôlables en ces matières. "La notion clef de mesure sous-tend les efforts entrepris dans cette voie, mais elle reste l"objet de débats confus ou peu concluants; la métrologie est, d'ailleurs, comme la statistique, une discipline délicate et d'accès difficile". Selon l'auteur, il semble préférable "en l"état actuel des Sciences Humaines, d'adopter le point de vue de la «dimensionnalisation du réel», qui s'appuie sur les données concrètes, plutôt que le point de vue de la «mathématisation du réel» qui repose sur une axiomatique métrologique". Il ajoute cependant que rien n'interdit aux spécialistes des Sciences Humaines de tirer parti de l'acquis métrologique des Sciences Exactes, en l'adaptant à leurs propres besoins dans un esprit interdisciplinaire (p. 211 ).

Il semble possible d'établir des correspondances structurales entre les processus génétiques conduisant à des structures naturelles et les démarches théoriques qui, procédant par abstraction réfléchissante et axiomatisation, débouchent précisément sur les mathématiques relationnelles et dynamiques, entre autres la théorie des ensembles, la topologie (les modèles mathématiques des structures, de treillis, de graphe orienté, etc.). C'est ainsi que les sciences psychosociologiques peuvent se livrer à une réduction directe des structures opératoires mentales ou de structures sociales à des structures implicatives logicomathématiques.

Applications au programme de la maîtrise en psychoéducation

Le point de vue explicité plus haut suppose donc une grande cohérence interne dans l'organisation même des connaissances à 
transmettre aux futurs psychoéducateurs. Cette transmission des connaissances comporte des modes de structuration multiples et sans cesse renouvelés. On ne peut donc procéder avec fruit à leur analyse que lorsqu'ils sont constitués ou, tout au plus, au cours de leur création. Ce point de vue «organiciste», dont le promoteur le plus convaincu est le théoricien Bertalanffy - qui a créé un mouvement de théorie générale des systèmes ${ }^{9}$ comporte les deux concepts-synthèses de structure et de fonction qui se retrouvent dans les systèmes d'ensemble.

L. Von Bertalanfly a affirmé que la Psychologie Américaine de la première moitié du vingtième siècle a été dominée par le concept de l'organisme réactif ou, de façon plus dramatique, par le modèle de l'homme conçu comme un robot. Cette conception rejoignait toutes les grandes écoles, classique et néo-béhavioriste, théories de l'apprentissage et de la motivation, psychanalyse, cybernétique, le concept du cerveau comme un ordinateur, et ainsi de suite. Cet auteur, considéré comme le principal pionnier de la théorie générale des systèmes, présente sa recherche dans General System Theory: Foundations, Development, Applications (N.Y : Braziller, 1968).

Un disciple de Bertalanffy travaillant au Master lnstitute of Technology est venu à l'École de Psychoéducation expliquer l'aspect de structure et de fonction du système d'ensemble. Un autre a fait connaître l'ordinateur au Centre d'orientation. En 1974, un ordinateur était accessible aux enfants pour les aider à apprendre à lire et à écrire. Cet instrument suscitait chez eux un intérêt très grand et facilitait leur apprentissage.

La conception épigénétique sera abondamment utilisée pour transmettre les connaissances. Le choix des genres de concepts

\footnotetext{
${ }^{9}$ Différents auteurs dont Bertalanfly font une revue critique de la théorie générale des systèmes dans Modem Systems Research for the Behavioral Scientist, édité par Walter Buckley chez Aldine Publishing Company Chicago en 1968.
}

utilisés sera aussi déterminé par le point de vue adopté, c'est-à-dire celui des concepts relationnels et fonctionnels subordonnant dès le point de départ «les éléments à des relations qui les englobent » et concevant réciproquement le tout comme le produit de la composition de ces interactions formatrices. Les concepts choisis seront aussi des concepts psychosociologiques intégrés qui permettront de présenter, d'une part, une conception intégrée et unifiée de l'individu, laquelle lorsque mesurée à l'évidence expérimentale et à l'observation quotidienne s'avère assez exacte et, d'autre part, une conceptualisation de l'entourage en interaction avec l'individu, c'est-à-dire «a whole world to be whole in ». Cette «intégration stéréoscopique » des deux théories fournies par Erikson et Piaget qui se complètent l'une l'autre aura toutes les chances de faire émerger une compréhension de «l'individu intégré dans un milieu », ce qui est essentiel aux psychoéducateurs cliniciens.

LE MODE DE STRUCTURATION DES CONNAISSANCES DANS LE PROGRAMME DE MAÎTRISE EN PSYCHOÉDUCATION REPOSE SUR LES TROIS QUESTIONS PRINCIPALES QUI, SELON PIAGET, SE RETROUVENT EN TOUTES LES BRANCHES DES SCIENCES DE L'HOMME.

IL S'AGIT :

1- Du développement ou de l'évolution dans le sens de la production graduelle de «formes organisées" avec transformations qualitatives au cours des étapes du développement de la rééducation;

Les quatre étapes du processus de rééducation du jeune délinquant (acclimatation, contrôle, production et personnalité) telles que décrites par Guindon dans sa thèse (p. 227-287) constituaient, de l'une à l'autre, des transformations qualitatives des formes organisées.

2-Du fonctionnement sous ses formes équilibrées ou synchroniques de ces mêmes étapes: par exemple, la différenciation, l'intégration et la consolidation sont des formes équilibrées et synchronisées de ces mêmes étapes.

3- Des échanges entre l'organisme et son milieu (milieu physique et autres organismes).

Paidéia, FFCLRP-USP, Rib. Preto, junho/99. 
ENN D'AUTRES TERMES, CES NOTIONS SONT CELLES DE:

1) La production de nouvelles structures dans la restructuration de la personnalité au cours du processus rééducatif: les acquis d'un individu rééduqué lui appartiennent et font partie de sa nouvelle structure, sa structure réorganisée.

2) L'équilibre, dans le sens de régulations et d'autoéquilibrations (unité fonctionnelle), par exemple: celui qui est parvenu à développer une autonomie corporelle est capable de vivre en harmonie avec son corps.

3) L'échange, dans le sens d'échanges matériels, de l'échange d'information, mais tout autant de l'échange affectif.

Ces productions, régulations et échanges se manifestent aussi bien sous les formes organiques que mentales ou intrapsychiques.

\section{LA MUTUALITÉ EST ABSOLUMENT NÉCESSAIRE DANS UN TEL PROCESSUS.}

\section{Références Bibliographiques}

Alain, J. (1993). La personne et son entourage. Sans Frontières les forces psychologiques, (8) 2, Montréal, IFHIM, p. 3 -13.

Desmarais, M.M. (1992). Un engagement social dans un projet collectif selon le processus d'actualisation des forces vitales humaines. La Vie sans frontières, no 2, Montréal, IFHIM, p. 27-32.

Guindon, J. (1969). Le processus de rééducation du jeune délinquant par l'actualisation des forces $\mathrm{du}$ moi. Contribution à l'étude des sciences de l'homme, no 7, Montréal, CRRH.

Guindon, J. (1982). Vers l'autonomie psychique de la naissance à la mort, Paris, Fleurus.

Guindon, J. (1987). L'identité de soi et ses facettes. Sans Frontières les forces psychologiques (2) 1, Montréal, IFHIM, p. 3 -23.

Guindon, J. (1988). Les étapes d'une phase de vie adulte. Sans Frontières les forces psychologiques, 3 (1), Montréal, IFHIM, p. 3 -23.

Paidéia, FFCLRP-USP, Rib. Preto, junho/99.
Guindon, J. (1992). La formation humaine intégrale et ses répercussions sociales. Sans Frontières les forces psychologiques, (7) 2, Montréal, IFHIM, p. $3-18$.

Sillamy, N, (1983). Dictionnaire usuel de psychologie, Paris, Bordas. 